\title{
Localization of Syntactic Comprehension by Positron Emission Tomography
}

\author{
Karin Stromswold,$* \dagger$ David Caplan, $*$ Nathaniel Alpert, $\ddagger$ \\ AND SCOTt RAUCh $\$$
}

\begin{abstract}
*Neuropsychology Laboratory, Department of Neurology, Massachusetts General Hospital, $\dagger$ Department of Brain and Cognitive Sciences, Massachusetts Institute of Technology, $\$$ Nuclear Medicine, Department of Radiology, Massachusetts General Hospital, and $\S$ Department of Psychiatry, Massachusetts General Hospital
\end{abstract}

Positron Emission Tomography (PET) was used to determine regional cerebral blood flow (rCBF) when eight normal right-handed males read and made acceptability judgments about sentences. $\mathrm{rCBF}$ was greater in Broca's area (particularly in the pars opercularis) when subjects judged the semantic plausibility of syntactically more complex sentences as compared to syntactically less complex sentences. rCBF was greater in left perisylvian language areas when subjects had to decide whether sentences were semantically plausible than when subjects had to decide whether syntactically identical sentences contained a nonsense word. The results of this experiment suggest that overall sentence processing occurs in regions of the left perisylvian association cortex. The results also provide evidence that one particular aspect of sentence processing (the process that corresponds to the greater difficulty of comprehending center-embedded than right-branching relative clause sentences) is centered in the pars opercularis of Broca's area. This process is likely to be related to the greater memory load associated with processing center-embedded sentences. () 1996 Academic Press, Inc.

The research reported here was supported by a grant from NIDCD (1RO3-DC01198) to D.C. K.S. was supported by an M.D./Ph.D. fellowship from the John D. and Catherine T. MacArthur Foundation, with additional support from NIDCD Grant 1RO3-DC01198 and a grant from the John Merck Scholars Program in the Biology of Developmental Disabilities in Children. S.R. was supported by the Postgraduate Program in Radiological Science (NCI Grant T32CAO93262). K.S. is now at the Department of Psychology and Center for Cognitive Science; Psychology Building, Busch Campus; Rutgers University; New Brunswick, NJ 08903 (karin@ ruccs.rutgers.edu). We thank Amy Biel, Marie Coppola, Joy Hanna, Betty Jaros, Lisa Torreano, and Nikos Makris for their assistance in subject testing and data analysis. We are also grateful for comments and suggestions made by Jane Grimshaw, Alec Marantz, Stacey Marsella, Jacques Mehler, Steven Pinker, Charles Schmidt, William Snyder, Anne Young, and three anonymous reviewers. Address correspondence to David Caplan, M.D., Ph.D., Neuropsychology Laboratory, Vincent Burnham 827, Massachusetts General Hospital, Fruit Street, Boston, MA, 02114. Fax: (617)726-2353; E-mail: Caplan@helix.mgh.harvard.edu. 


\section{INTRODUCTION}

Until recently, essentially all that was known about the neural basis of language came from studies of aphasic patients. The intact cognitive abilities of some aphasic patients suggest that the neural structures responsible for language are distinct from those responsible for nonlanguage tasks (Caplan, 1987, 1992; Shallice, 1988). In addition, the relatively selective linguistic deficits of some aphasic patients (e.g., patients with impaired phonological or naming abilities but intact grammatical abilities) are consistent with there being distinct neural structures responsible for different aspects of language (Shallice, 1988). Despite these findings, attempts to reliably link particular brain regions to particular linguistic processes have yielded mixed results.

Within the domain of sentence processing, patients have been described who have difficulty constructing syntactic structures in comprehension tasks and/or in using these structures to determine the propositions expressed in sentences (Caramazza \& Zurif, 1976; Caplan, Baker, \& Dehaut, 1985; Caplan \& Futter, 1986; Caplan \& Hildebrandt, 1988; Linebarger, Schwartz, \& Saffran, 1983; Schwartz, Linebarger, Saffran, \& Pate, 1987; Tyler, 1985; Zurif, Swinney, Prather, Solomon, \& Bushell, 1993). Many of these patients are agrammatic Broca's aphasics, a fact that has led several researchers to suggest that Broca's area is the most important part of a neural net that is responsible for syntactic processing (Mesulam, 1990; Damasio, 1992). However, lesions in patients with Broca's aphasia often extend well beyond Broca's area (Mohr, Pessin, Finkelstein, Funkenstein, Duncan, \& Davis, 1978; Selnes, Knopman, Niccum, Rubens, \& Larson, 1983; Vanier \& Caplan, 1990). Furthermore, patients with aphasic syndromes other than agrammatic Broca's aphasia, whose lesions are likely to involve or be restricted to regions other than Broca's area, often show impairments of syntactic comprehension (Caplan et al., 1985; Caplan \& Hildebrandt, 1988; Tramo, Baynes, \& Volpe, 1988). Thus, the hypothesis that Broca's area is the sole area responsible for parsing, or for a particular set of parsing operations, can only be said to receive modest support from lesion-deficit correlational studies of aphasic patients.

In addition to studies of aphasic patients, recently a number of techniques have been used to measure the brain activity associated with language processing in normal adults. Although event-related potentials (ERPs) have proved quite useful in characterizing the time course of various aspects of linguistic processing (see, e.g., Munte, Heinze, \& Mangun, 1993; Neville, Nicol, Barss, Forster, \& Garrett, 1991; Rosler, Putz, Friederici, \& Hahne, 1993), ERP studies lack the necessary spatial resolution to be useful in determining the neural sites of language processing. In the past 6 years, positron emission tomography (PET) has emerged as a powerful new technique for localizing the neural structures responsible for components of language pro- 
cessing. Thus far, most PET studies have been concerned mainly with delineating regions of the brain that are associated with phonetic and phonological processing (e.g., Demonet, Chollet, Ramsay, Cardebat, Nespoulous, Wise, Rascol, \& Frackowiak, 1992; Paulesu, Frith, \& Frackowiak, 1993; Petersen, Fox, Posner, Mintun, \& Raichle, 1989; Sergent, Zuck, Levesque, \& MacDonald, 1992; Zatorre, Evans, Meyer, \& Gjedde, 1992) or with the recognition, comprehension, and production of single words (e.g., Demonet et al., 1992; Frith, Friston, Liddle, \& Frackowiak, 199; Friston, Frith, Liddle, \& Frackowiak, 1991; Petersen, Fox, Posner, Mintun, \& Raichle, 1988; Petersen et al., 1989; Petersen, Fox, Snyder, \& Raichle, 1990; Posner, Petersen, Fox, \& Raichle, 1988; Sergent et al., 1992; Wise, Chollet, Hadar, Friston, Hoffner, \& Frackowiak, 1991).

Recently, PET has been used to investigate the regional brain activity associated with sentence-level language processing. Mazoyer and his colleagues (Mazoyer, Tzourio, Frak, Syrota, Murayama, Levrier, Salamon, Dehaene, Cohen, \& Mehler, 1993) compared the rCBF when native speakers of French were at rest with the $\mathrm{rCBF}$ when they listened to stories in a foreign language (Tamil), lists of French words, a French story in which every content word was replaced with a pseudoword (pseudoword condition), a French story in which every content word was replaced with a semantically unrelated word from the same grammatical category (semantic anomaly condition), and a story in French. Mazoyer et al. (1993) hypothesized that, for French speakers, listening to a Tamil story would result in only acoustic processing. Listening to lists of French lexical or content words would result in acoustic, phonological, and lexical processing. Listening to a pseudoword story was predicted to result in acoustic, phonological, prosodic, and syntactic processing. Mazoyer et al. (1993) further hypothesized that subjects would acoustically, prosodically, lexically, and syntactically process both the semantically anomalous French story and the good French story, but only the good French story would cause the subject to engage in "conceptual processing." Unfortunately, the results of Mazoyer et al. indicate that subjects did not process all of the stimuli as the experimenters predicted they would. For example, the scanning results suggest that the subjects lexically processed the words in the French word list and the French story but not the words in the semantically anomalous French story. Furthermore, Mazoyer et al. (1993) did not detect consistent regional activity associated with syntactic, prosodic, or conceptual processing. They interpreted their failure to do so as evidence that the "speech processing system of the human brain is not organized, at the neural level, in a hierarchy of areas that successively and automatically come into play whenever they receive an adequately structured stimulus. Rather, speech processing seems to imply the coordination of a network of areas, each of which may be specialized in one aspect of speech processing, but require coherent support from the others in order to reach a high level of activation", 
(Mazoyer et al., 1993, p. 476). Although this explanation might be correct, a plausible alternative is that the semantically anomalous and pseudoword conditions were aberrant enough that subjects did not process them as fully as Mazoyer et al. (1993) predicted, and thus, these conditions did not differ in the minimal ways necessary to isolate the neural correlates of the various components of linguistic processing above the single-word level.

Another study involving sentences has been reported by Bookheimer, Zeffiro, Gallard, and Theodore (1993). Bookheimer et al. (1993) had subjects judge whether sentences were the same in meaning when they contained the same words but differed in word order (e.g., The lake is west of the city; West of the city is the lake) and when they had the same syntax but differed in one word. In three control conditions, subjects monitored sentences for a phoneme change, heard identical pairs of sentences, and rested. Based on a series of subtractions, the authors concluded that syntax processing increased rCBF in Broca's area and in the left hippocampus. However, the comparison of the syntax conditions with the baseline conditions is far from pure: the syntax task involves reasoning and other operations (e.g., verbal short-term memory) that are not required in any of the baseline conditions and, conversely, the baseline conditions may involve operations (such as sustaining attention in the phoneme change monitoring task) that are not found in the experimental syntax stimulation conditions.

These two studies provide conflicting data regarding the localization of syntactic processing; indeed, the areas that are implicated in syntactic processing are completely non-overlapping in the two studies. However, for the reasons discussed above, the experimental designs and behavioral results of these two studies are such that neither cleanly isolated syntactic processing or sentence processing. The two studies both suffer from problems that have been identified in PET language studies (e.g., see recent critiques by Demonet, Wise, \& Frackowiak, 1993; and Sergent et al., 1992). These are: (1) the inadequate appreciation of all of the task demands associated with experimental conditions; (2) the use of experimental conditions that are meant to be minimally different, but are not; (3) the use of experimental conditions that are not well-motivated on either psycholinguistic or linguistic grounds; (4) the lack of any independent way of determining whether subjects are actually performing the task (or are performing the task in the expected manner); and (5) problems associated with the presentation of stimuli in blocked format for extended periods of time.

In the following study, we used PET to measure the regional brain activity that is present when normal subjects read and judge the acceptability of sentences. By using a tightly controlled experimental design and making measurements of subjects' sentence-processing functions while they were being scanned, we increase the likelihood of identifying the regions involved in various aspects of sentence processing. 


\section{Condition 1}

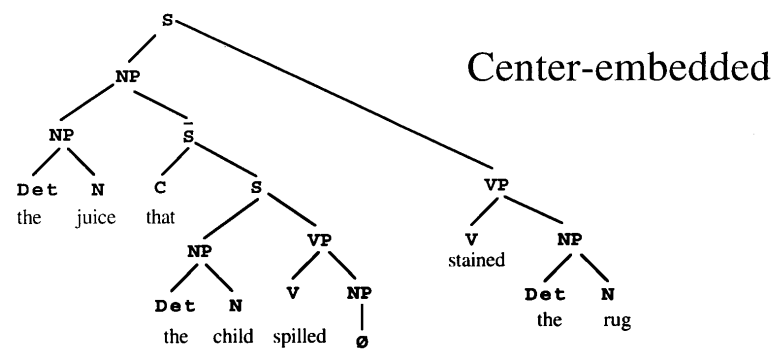

Center-embedded construction: The juice that the child spilled _ stained the rug

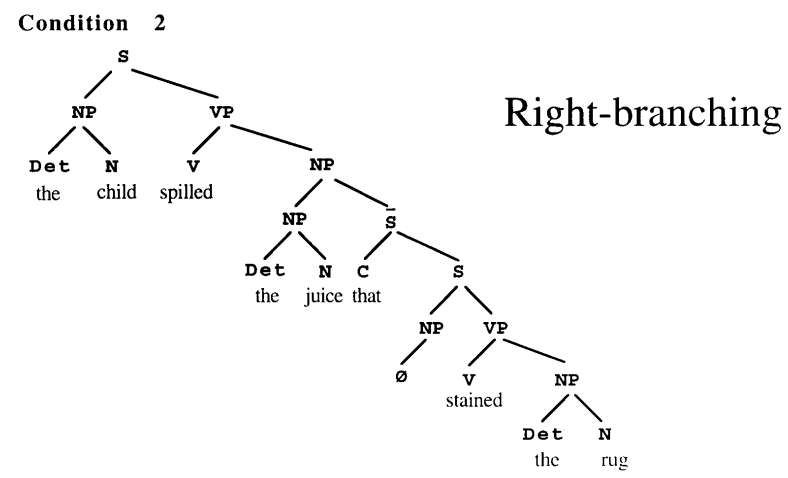

Right branching construction: The child spilled the juice that _ stained the rug

FIG. 1. Tree diagrams for right-branching and center-embedded relative clause constructions.

\section{EXPERIMENT}

Subjects. Eight monolingual native English-speaking male college students (ages 19-28) participated. All were strongly right-handed and had no first-degree left-handed relatives. All had normal vision and hearing and no positive neurological or psychiatric history.

Conditions. Subjects were scanned under three experimental conditions. Sentences in condition 1 contained center-embedded relative clauses and sentences in condition 2 contained right-branching relative clauses (see Fig. 1). In conditions 1 and 2 , the sentences contained verbs that required that a noun in either subject or object position be either animate or inanimate. Half of the sentences in conditions 1 and 2 were semantically plausible sentences that obeyed this restriction (e.g., the center-embedded sentence The juice that the child spilled stained the rug, and the right-branching sentence The 
child spilled the juice that stained the rug), and half were semantically implausible sentences that violated this restriction (e.g., the center-embedded sentence *The child that the juice spilled stained the rug or the rightbranching sentence *The juice stained the rug that spilled the child). ${ }^{1}$

Half of the sentences in condition 3 were exactly the same as the acceptable (plausible) center-embedded and right-branching relative clause used in conditions 1 and 2. The other half of the sentences were unacceptable because one of the nouns or verbs had been replaced with a orthographically and phonetically possible pseudoword (e.g., *The juice that the child chorried stained the rug, and *The child spilled the juice that mulved the rug).

In both condition 1 and condition 2 , in order to decide whether a sentence is acceptable, a subject must parse and interpret the sentence. Rightbranching and center-embedded sentences were chosen as stimuli in this experiment because results from previous psycholinguistic research indicate that normal subjects reliably make more errors and take longer to process sentences that contain center-embedded relative clauses sentences than sentences that contain right-branching relative clauses (e.g., Caplan, Hildebrandt, \& Waters, 1994; King \& Just, 1991; Waters, Caplan, \& Hildebrandt, 1987). Although the exact reason subjects have more difficulty processing center-embedded than right-branching sentences is not clear, it is often thought to result from the memory load associated with holding the matrix subject NP in a parsing buffer until it is assigned a thematic role (Berwick \& Weinberg, 1984; Marcus, 1980; Wanner \& Maratsos, 1978), or with the combination of memory load and the operation of structuring the relative clause (Just \& Carpenter, 1992). Thus, if PET scanning reveals that the rCBF for a particular region is greater in condition 1 than condition 2, this region may be an important part of the neural system responsible for the type of memory that is particularly taxed by the processing of center-embedded relative clauses.

Because half of the sentences in condition 3 were identical to the acceptable sentences in conditions 1 and 2 and half of the sentences differed by the presence of a single pseudoword, condition 3 is a control condition for conditions 1 and 2. Logically, in order to correctly decide whether a sentence in condition 3 does or does not contain a pseudoword, a subject must minimally read each word in a sentence and determine if it is a real English word. If subjects judged the sentences in condition 3 without syntactically parsing or interpreting the sentences, then differences in the PET activity associated with condition 3 and the PET activity in conditions 1 or 2 could arguably be said to correspond to regions that are associated with the processes of sentence parsing and interpretation. However, the behavioral data collected

${ }^{1}$ Adopting the linguistic convention, an asterisk (*) will be used to indicate that a sentence is unacceptable. Depending on the context, an asterisk may indicate that a sentence is semantically implausible or that a sentence contains a non-English word. 
TABLE 1

Sentence types used in conditions 1-3

Condition 1: Center-embedded sentences

Acceptable

Anomalous
The limerick that the boy recited appalled the priest

The teenager that the miniskirt wore horrified the mother

Condition 2: Right-branching sentences

Acceptable

Anomalous

The biographer omitted the story that insulted the queen

The woman tipped the barber that pleased the haircut

Condition 3: Sentences with nonwords

Acceptable

Unacceptable
As in tasks 1 and 2

The economist predicted the recession that chorried the man The sculpture that the artist exhibited shocked the findle

Language functions thought to be assessed in PET subtractions

\begin{tabular}{|c|c|c|c|}
\hline Comparison & Stimulated state & Baseline state & Cognitive operation \\
\hline Condition $1-2$ & $\begin{array}{l}\text { Center-embedded } \\
\text { sentences }\end{array}$ & $\begin{array}{l}\text { Right-branching } \\
\text { sentences }\end{array}$ & Syntactic processing \\
\hline Condition $1-3$ & $\begin{array}{l}\text { Center-embedded } \\
\text { sentences }\end{array}$ & $\begin{array}{l}\text { Sentences with } \\
\text { nonwords }\end{array}$ & $\begin{array}{l}\text { Semantic processing } \\
\text { of sentences }\end{array}$ \\
\hline Condition $2-3$ & $\begin{array}{l}\text { Right-branching } \\
\text { sentences }\end{array}$ & $\begin{array}{l}\text { Sentences with } \\
\text { nonwords }\end{array}$ & $\begin{array}{l}\text { Semantic processing } \\
\text { of sentences }\end{array}$ \\
\hline
\end{tabular}

during scanning suggest that subjects did syntactically parse the sentences in condition 3, even though, logically, it was not required (see Behavioral Results and Discussion sections). If subjects did, in fact, syntactically process sentences in condition 3 , then brain regions which are more active in conditions 1 or 2 than in condition 3 could arguably be said to be primarily those involved in aspects of sentence processing other than syntactic processing, such as assigning the meaning of a sentence or determining whether a sentence is plausible.

Table 1 presents a summary of the stimuli used in each condition and the psycholinguistic processes we tentatively suggest are associated with the subtractions performed.

The temporal resolution of PET is such that, if one wants to study the regions of the brain that are associated with a particular task, one must have subjects perform the task in question for an extended period of time. In previous psycholinguistic experiments in which subjects were asked to process center-embedded and right-branching relative clause sentences, the two types of sentences were presented together in random order. To establish whether the blocked presentation required by the temporal resolution of PET affects processing of center-embedded and right-branching sentences, 19 righthanded, monolingual English-speaking college students judged the semantic plausibility of center-embedded and right-branching sentences that were presented in blocked or mixed format. Analysis of variance of the reaction time 
data revealed that blocking had no effect on subjects' ability to make plausibility judgments. ${ }^{2}$

Stimulus and experimental design. A number of controls and counterbalances were introduced to ensure that the three conditions differed only on the dimension(s) outlined above, and to ensure that subjects did not adopt alternative strategies for judging the sentences. The following factors were controlled for in the design of the stimuli and the experiment.

1. Sentences were based on scenarios. There were a total of 144 scenarios (such as the scenario involving a child staining a rug by spilling juice onto it) and each scenario appeared in each condition equally often across subjects. Because each scenario appeared in each condition equally often, differences in semantic goodness of scenarios, frequency of words, word choice, etc., could not be responsible for any differences in rCBF between the conditions. No verb appeared in more than one scenario and no subject judged any scenario more than twice.

2. The animacy of subject and object noun phrases and the plausibility of the sentences varied orthogonally. Thus, for example, the semantically plausible sentence The patient that the drug cured thanked the doctor and the semantically implausible sentence *The girl that the miniskirt wore horrified the mother both contained an animate noun phrase, followed by an inanimate noun phrase, followed by an animate noun phase. Animacy type, acceptability, and sentence type were counterbalanced within subjects. This feature of the design was included to ensure that subjects could not make plausibility judgments on the basis of the sequence of animacy of the nouns.

3. All noun phrases were singular, common, and definite. This feature of the design was included to ensure that subjects would not be influenced by discourse effects.

4. In conditions 1 and 2 , sentences became implausible at various points in the relative clauses and the main clauses. This feature was included to ensure that subjects had to read the sentences in their entirety before they could decide if it was plausible. Overall, the point at which center-embedded sentences became implausible was earlier than the point at which right-branching sentences became implausible. This feature was included

${ }^{2}$ A 2 (blocked vs. mixed) $\times 2$ (center-embedded vs. right-branching) $\times 2$ (plausible vs. implausible) analysis of variance was performed on the reaction time data for the sentences that were judged correctly. There was a significant main effect of sentence type $(F(1,18)=$ $9.30 ; p=.007)$, with center-embedded sentences taking an average of $236 \mathrm{msec}$ longer than right-branching sentences to judge. There was no effect of blocking $(F(1,18)=2.2$, ns) and no interaction of blocking with any other variable. Mean RTs for center embedded sentences were $3248 \mathrm{msec}$ in the blocked condition and $3349 \mathrm{msec}$ in the mixed condition; mean RTs for right-branching sentences were $2930 \mathrm{msec}$ in the blocked condition and $3194 \mathrm{msec}$ in the mixed condition. 
to eliminate the possibility that the advantage enjoyed by right-branching sentences was attributable to right-branching sentences becoming implausible at an earlier point than center-embedded sentences.

5. In condition 3, with the exception of the first noun, which was never replaced, each noun and verb was replaced equally often with a pseudoword. This feature was included to encourage subjects to read each sentence in its entirety before deciding that the sentence did not contain a pseudoword.

6. The three conditions were presented in blocked format, with each subject being presented each condition twice. Plausibility blocks contained 24 trials, and pseudoword blocks contained 48 trials. The order of presentation of blocks (conditions 1,2, and 3) was counterbalanced across subjects in order to eliminate any effect of order of presentation on behavioral or PET data.

Behavioral testing procedure. PET scans were taken as subjects read and judged the goodness of sentences presented visually in whole sentence format on a Macintosh Classic II computer screen. The computer screen rested on a shelf approximately 12 inches from the subject's eyes. After a 300msec fixation point, a whole sentence appeared on a single line, subtending a visual angle of $20-25^{\circ}$. This sentence remained on the computer screen until the subject responded via keypresses with two fingers of the left hand. After a response, the screen was blank for $700 \mathrm{msec}$, followed first by the 300-msec fixation point, and then by the next sentence to be judged. Reaction time and error rate data were collected during PET scanning and subjects were told to make acceptability judgments as quickly as possible without making errors.

At the beginning of the experiment, subjects were given six practice trials judging simple active sentences for semantic plausibility (e.g., The child licks the lollipop, *The lollipop licks the child) or the presence of a pseudoword (e.g., The child licks the lollipop, *The child vugs the lollipop). At the beginning of each block, subjects were told whether they would be making pseudoword or plausibility judgments in that block.

\section{PET PROCEDURES}

Image collection. A General Electric Scanditronix PC4096 15 slice wholebody tomograph was used in its stationary mode to acquire PET data in contiguous slices with center-to-center distance of $6.5 \mathrm{~mm}$ (axial field equal to $97.5 \mathrm{~mm}$ ) and axial resolution of $6.0 \mathrm{~mm}$ FWHM, with a Hanning-weighted reconstruction filter set to yield 8.0-mm in-plane spatial resolution (FWHM). The studies were carried out in the MGH PET imaging suite which has been designed to provide for control of ambient light, temperature, and noise level. Subjects' heads were restrained in a custom-molded thermoplastic face mask, and aligned relative to the cantho-meatal line, using horizontal and vertical 
projected laser lines. Subjects inhaled ${ }^{15} \mathrm{O}-\mathrm{CO}_{2}$ gas by nasal cannulae within a face mask for $90 \mathrm{sec}$, reaching terminal count rates of 100,000 to 200,000 events per second. Previous work in our laboratory has demonstrated that the integrated counts over inhalation periods up to $90 \mathrm{sec}$ are a linear function over the flow range 0 to $130 \mathrm{ml} / \mathrm{min} / 100 \mathrm{~g}$ (unpublished data).

Image reconstruction. Each PET data acquisition run consisted of 20 measurements, the first 3 with $10 \mathrm{sec}$ duration each and the remaining 17 with $5 \mathrm{sec}$ duration each. Scans 4-16 were summed after reconstruction to form images of relative blood flow. The scan data were pooled, by slice over all runs, to form images of higher statistical quality. These images were examined visually and the coordinates of midline structures across all slices were recorded and used in a least squares fitting procedure to estimate the parameters of the midsagittal plane. Transverse section images of all the emission and transmission scans were resliced parasagittally at 5.1-mm intervals. The brain surface of a 10.2-mm parasagittal slice was manually outlined at the $50 \%$ threshold level. Surface data missing from the parasagittal emission slice were filled in from the more complete sagittal transmission images when necessary. Parameters for the transformation of PET data to the coordinate system of Talairach were obtained by deforming the 10 -mm sagittal plane from the 1967 Talairach brain atlas (Talairach \& Szikla, 1967), using a nonlinear fitting procedure that included three magnifications along the anterior commissure-posterior commissure (AC-PC) line and one vertical magnification to match the location of the frontal pole, occipital pole, vertex, anterior, and posterior commissures and the tilt angle in a least-squares algorithm (Alpert, Berdichevsky, Weise, Tang, \& Rauch, 1993). Quality of the fitting procedure was judged both by the standard errors of the parameters and by visual comparison of the manually drawn brain surface and the atlas contour. A computerized version of the 1967 Talairach atlas showing ROIs was projected onto the transformed PET data with a digitized mapping program (Alpert et al., 1993).

Hypotheses. We based a priori predictions on the results of lesion-deficit correlational studies that support the view that syntactic processing primarily involves Broca's area (Mesulam, 1990; Damasio, 1992). Accordingly, we predicted that center-embedded sentences would engage Broca's area significantly more than would right-branching sentences. Based on the results of lesion-deficit correlational studies that show that many aspects of sentence processing are disrupted by lesions throughout the perisylvian cortex, we made the a priori prediction that making semantic plausibility judgments of sentences would engage all parts of the left perisylvian association cortex (including Broca's area) significantly more than would making pseudoword judgments.

Statistical analyses. The mean concentration in each run was obtained as an area-weighted sum of the concentration of each slice and normalized to a nominal value of $50 \mathrm{ml} / \mathrm{min} / 100 \mathrm{~g}$. The data were then rescaled, smoothed with a two-dimensional Gaussian filter of width $20 \mathrm{~mm}$ (FWHM), pooled 
over all subjects for each scanning condition, and subtracted pairwise within subjects and between tasks to yield images of mean difference, standard deviation, $N$ (number of data points contributing to each pixel), and $t$ statistic, as well as omnibus subtraction images (OSIs; with values in $z$ score units) as described by Friston et al. (1991). Statistical analysis and hypothesis testing followed Friston et al. (1991). Two analyses were performed: (1) ROI analyses were performed on the PET data with region boundaries generated by the mapping program to test our a priori hypothesis, and (2) post hoc review of the OSIs were performed for the whole brain in search of additional regions of statistically significant activation. In both types of analyses, OSIs of experimental minus control tasks were used. The brain volumes for all ROIs tested were defined on the basis of the digitized version of the 1967 Talairach atlas. Each region so identified provides an objective means for adjusting the significance level of the SPM according to the number of voxels tested.

\section{BEHAVIORAL RESULTS}

Subjects were extremely accurate in their judgments about the acceptability of sentences, making only 59 errors out of a total of 1536 trials. RT data for correctly judged sentences were analyzed using a 2 (center-embedded vs. right-branching) $\times 2$ (semantic plausibility vs. pseudoword judgment) $\times 2$ (first block vs. second block) $\times 2$ (good vs. bad) analysis of variance. There was a significant main effect for the type of sentence structure, $F(1$, $7)=16.78, p=.005$, with subjects requiring an average of $3422 \mathrm{msec}$ to judge center-embedded relative clause sentences and $3033 \mathrm{msec}$ to judge right-branching relative clause sentences. There was also a significant main effect for the type of judgment made, $F(1,7)=33.39, p=.001$, with plausibility judgments (condition 1 and 2) taking an average of $3972 \mathrm{msec}$ and pseudoword judgments (condition 3) taking an average of $2483 \mathrm{msec}$. The only other significant effect or interaction was a significant interaction between type of judgment and good versus bad sentences, $F(1,7)=40.35, p$ $<$.0005), with subjects, on average, requiring $370 \mathrm{msec}$ more to reject an implausible sentence than to accept a plausible sentence and $442 \mathrm{msec}$ less to reject a sentence that contained a pseudoword than to accept a sentence that contained only legitimate English words. Neither the two-way interaction between clause type (center-embedded versus right-branching) and judgment type (plausibility versus pseudoword), nor the three-way interaction among clause type, judgment type, and goodness were significant $(p>.2$ and $p>.8$, respectively).

Because block number neither had a significant effect nor entered into any significant interactions, further analyses collapsed across block number. Separate 2 (clause type) $\times 2$ (good vs. bad) analyses of variance were performed on just the plausibility data (conditions 1 and 2) and just the pseudoword data (condition 3). These analyses revealed that the differences in RTs for good versus bad sentences were significant for both the plausibility 
data $(F(1,7)=6.79, p=.035)$ and the pseudoword data $(F(1,7)=24.81$, $p=.002)$. For the plausibility conditions, differences in the syntax of the sentences (center-embedded vs. right-branching) had a significant effect on reaction time, $F(1,7)=7.77, p=.027$, with subjects requiring $4230 \mathrm{msec}$ to judge center-embedded sentences and $3719 \mathrm{msec}$ to judge right-branching sentences. Analysis of the reaction time data for pseudoword judgments (condition 3) also revealed a significant effect of syntax of the sentences, $F(1,7)=16.54, p=.005$, with subjects requiring on average $2612 \mathrm{msec}$ to judge center-embedded sentences and $2356 \mathrm{msec}$ to judge right-branching sentences. The significant effect of relative clause type was found for both the sentences in condition 3 that did contain a pseudoword $(F(1,7)=6.24$, $p=.041)$ and those that $\operatorname{did} \operatorname{not}(F(1,7)=11.04, p=.013)$.

\section{PRELIMINARY DISCUSSION OF BEHAVIORAL RESULTS}

The effect of sentence type in conditions 1 and 2 is expected and is attributable to syntactic processing or related functions (see General Discussion). The magnitude of the sentence type effect was comparable in size and direction with results reported in the psycholinguistic literature (e.g., Waters et al., 1987) and results obtained with the same stimuli on unscanned subjects (see footnote 2), suggesting that scanning did not affect the way subjects performed the tasks. The effect of condition was also expected. It indicates that determining that a sentence is semantically plausible requires greater amounts of processing than deciding whether all of its words are legitimate English words.

The finding of a sentence type effect in condition 3 is noteworthy. In condition 3, subjects had to decide whether a sentence contained a pseudoword. This only requires that one look up each word in the sentence and confirm that each word is present in the mental lexicon; it does not require that one either syntactically parse or semantically interpret the sentences. If subjects did not syntactically parse sentences when asked to make pseudoword judgments, then they should have been equally fast in judging the center-embedded and right-branching sentences in condition 3 . The fact that sentence type effects were found in the pseudoword judgment condition-and that they were equivalent to those found in the plausibility judgment conditions (conditions 1 and 2)-indicates that subjects assigned syntactic structure to the sentences in this condition. The faster RTs in the pseudoword judgment condition (condition 3 ) than in the plausibility judgment conditions (conditions 1 and 2) thus must be attributed to factors other than the presence of parsing in the plausibility judgment conditions and its absence in the pseudoword judgment condition (see General Discussion). ${ }^{3}$

${ }^{3}$ The finding that subjects parsed sentences in condition 3 is consistent with the claim in Fodor's (1983) modularity theory that parsing is obligatory when a sentence-like stimulus is presented. 
TABLE 2

Areas of Increased rCBF in Omnibus Statistical Images

\begin{tabular}{cccccc}
\hline & \multicolumn{3}{c}{ Threshold } & & \\
Max & for & Number of & Computed & Location \\
Region & $z$ score & $p<.05$ & pixels & $p$ value & $(\mathrm{X}, \mathrm{Y}, \mathrm{Z})$ \\
\hline
\end{tabular}

Analyses based on a priori hypotheses

Subtraction of condition 2 from 1
L. Broca's area
2.7
2.4
$131^{a}$
$<.025$
$46.5,9.8,4.0$
L. pars opercularis
2.7
1.8
$48<.008$
$46.5,9.8,4.0$

Subtraction of condition 3 from 1
L. Broca
2.8
2.4
$148^{a}$
$<.025$
$37.6,20.0,8.0$
L. superior temporal
3.0
2.0
70
$<.007$
$63.1,-18.2,8.0$
L. Wernicke's
2.6
1.9
$60<.015$
$47.8,-46.3,20.0$

Subtraction of condition 3 from 2
L. Broca's
2.8
2.4
$\begin{aligned} 135^{a} & <.02 \\ 56 & <.02\end{aligned}$
$50.4,13.6,16.0$
L. supramarginal
2.4
$56.7,-31.0,20.0$

Post hoc analyses of individual slices of the whole brain Subtraction of condition 3 from 1
Superior frontal area
3.8
3.65
$>3000$
.03
$2.0,30.0,48.0$

${ }^{a}$ The different number of pixels reflect the size of Broca's area in the different slices.

\section{rCBF RESULTS}

Table 2 shows the location of significant increases in rCBF based on statistical parameter mapping derived by subtracting PET activity in the plausibility judgment condition with right-branching sentences (condition 2) from the plausibility judgment condition with center-embedded sentences (condition 1), and by subtracting PET activity in the pseudoword judgment condition (condition 3) from each of the plausibility judgment conditions (conditions 1 and 2).

For comparisons of ROIs designated in the a priori hypotheses, there were significant increases in $\mathrm{rCBF}$ in several areas within the perisylvian association cortex of the left hemisphere. For the subtraction of condition 2 (rightbranching) from condition 1 (center-embedded), a significant increase in rCBF occurred only in Broca's area, with the center of activation in the pars opercularis (Fig. 2). The subtraction of the pseudoword condition (condition 3 ) from the center-embedded plausibility condition (condition 1) yielded significant increases in rCBF in Broca's area, Wernicke's area, and the adjacent portions of the superior temporal gyrus. The subtraction of the pseudoword condition (condition 3 ) from the right-branching plausibility condition (condition 2) yielded significant increases in Broca's area and the supramarginal gyrus.

The post hoc review of the omnibus subtraction images for the whole 


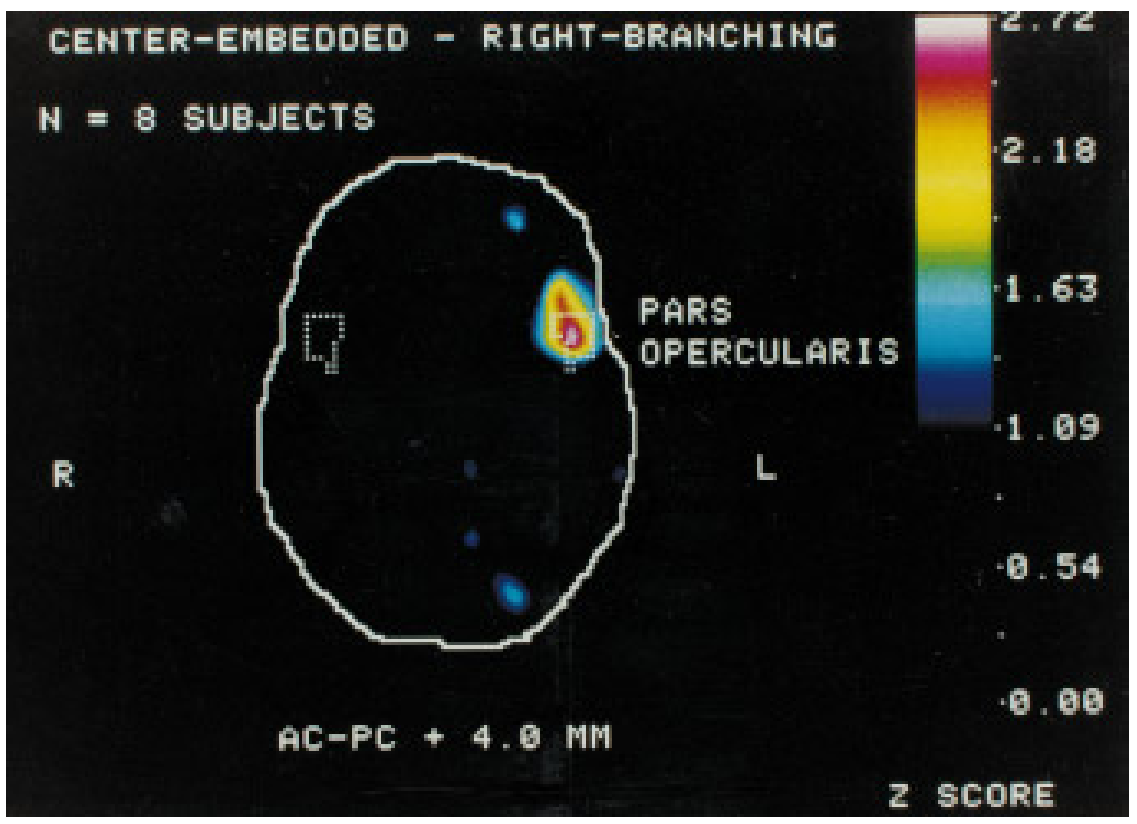

FIG. 2. Statistical parameter map (omnibus subtraction image) showing increased rCBF in the pars opercularis of the left hemisphere during judgments of semantic plausibility of sentences with center-embedded compared to right-branching relative clauses (condition 1 condition 2).

brain identified only a single significant increase in the rCBF. This increase in $\mathrm{rCBF}$ occurred in the subtraction of the pseudoword condition (condition 3 ) from the center-embedded plausibility condition (condition 1), and was located near the midline of the brain in the region of the frontal eye fields in the superior frontal lobe. Three other changes in $\mathrm{rCBF}$ produced $z$ scores of 3 or greater. These three changes occurred in the subtraction of the rightbranching plausibility condition (condition 2) from the center-embedded plausibility condition (condition 1 ), and were located close to the midline in the frontal eye fields $(z=3.0)$, close to the midline in the anterior cingulate gyrus $(z=3.6)$, and in the right middle temporal gyrus $(z=3.5)$. In the subtraction of the right-branching plausibility condition (condition 2) from the center-embedded plausibility condition (condition 1 ), there were no $z$ scores greater than 2.5 within the perisylvian cortex, other than the one seen in the pars opercularis that was mentioned above.

In order to characterize the reliability of the location of the activation found in Broca's area (pars opercularis) in the subtraction of condition 2 from condition 1, we performed an additional SPM analysis using a jackknife procedure (Mostellar and Tukey, 1977). The jackknife was formed by deleting subjects one at a time from the cohort, and computing eight SPMs. A 
ROI of $537 \mathrm{~mm}^{2}$ was drawn surrounding the activation and used to compute a $z$-centroid for each of the eight SPM's. The sample variance of the centroid was used to compute a $95 \%$ confidence limit for the location of activation. For pars opercularis $(Z=4.0 \mathrm{~mm})$ this was $X=+45.2 \pm 2.0 \mathrm{~mm} Y=$ $+15.2 \pm 2.4 \mathrm{~mm}$. This implies that this region was activated in a similar fashion in all subjects.

\section{DISCUSSION}

We first consider the results of the post hoc analyses of the OSIs for the whole brain. There was an increase in $\mathrm{rCBF}$ in the superior frontal lobe, near the midline of the brain in the region of the frontal eye fields, in the subtraction of pseudoword judgments (condition 3) from plausibility judgments for center embedded sentences (condition 1). This increase in rCBF may be due to differences in eye movements in the two conditions. For example, subjects might have gone back and reread portions of the sentences in condition 1 . Though this hypothesis cannot be proven on the basis of the data from the present experiment, it can be tested. If it is correct, there should be differences in eye movements in the relevant conditions that are measurable using ENGs or eye-trackers. In addition, if the hypothesis is correct, these $\mathrm{rCBF}$ effects should disappear when stimuli are presented auditorily.

In the subtraction of the right branching plausibility condition from the center embedded plausibility condition (condition 1-2), there were $z$ scores of 3 or greater in the SPM analysis in the frontal eye fields, the anterior cingulate gyrus, and the right middle temporal gyrus. Though these increases did not meet the SPM criteria for significance, their magnitude is sufficiently large that they merit consideration. The increase in $\mathrm{rCBF}$ in the frontal eye fields may reflect differences in eye movements in these conditions. The increased $\mathrm{rCBF}$ in the anterior cingulate gyrus may reflect changes in arousal and in attention. Center-embedded sentences are the most demanding stimuli that had to be processed in this experiment, as shown by the RT results. An increase in activation has often been found in the anterior cingulate when PET activity associated with a less complex task is subtracted from that associated with a more complex task, and has been attributed to attentional and arousal processes (Posner et al., 1988). Techniques that focus on the role of attentional factors have recently been reported for PET studies (Chertkow, Bub, Waters, Evans, Whitehead, \& Hosein, 1993), and may be useful in investigating this issue. The increase in $\mathrm{rCBF}$ found in the right middle temporal gyrus remains to be explained.

The analyses based upon the a priori predictions showed that these predictions were largely confirmed. The subtraction of the pseudoword condition (condition 3) from either of the plausibility judgment conditions (either condition 1 or condition 2) led to increased $\mathrm{rCBF}$ in several areas of the left perisylvian cortex, while the subtraction of the right branching plausibility 
condition (condition 2) from the center-embedded plausibility condition (condition 1) led to increased $\mathrm{rCBF}$ in part of Broca's area.

The increase in $\mathrm{rCBF}$ in various parts of the perisylvian association cortex associated with the subtraction of pseudoword condition (condition 3) from either of the plausibility judgment conditions (either condition 1 or condition 2) could be related to one or more of several differences between these conditions. As noted above, the presence of the same magnitude of sentence type effects in the pseudoword condition (condition 3 ) as in the plausibility judgment conditions (conditions 1 and 2) rules out the possibility that subjects parsed sentences in the plausibility judgment conditions and not in the pseudoword condition. The change in rCBF may reflect the presence of sentencelevel semantic processing in plausibility judgments and not in the pseudoword condition. If this is the case, the results suggest that several areas of the perisylvian cortex are involved in determining sentence meaning or in searching for a proposition in semantic memory. It should be noted that, if this is the correct interpretation of these results, Broca's area would appear to be involved in these processes, as well as in processes related to syntactic analysis. However, the implications of these results must be viewed extremely cautiously, because there is another account of the differences between the pseudoword condition and the plausibility judgment conditions simply in terms of strategies that subjects may have adopted in this experiment. It is possible that subjects processed the sentences containing pseudowords in the pseudoword condition (condition 3) only until a nonword was definitively recognized, whereas they continued to process all sentences in the plausibility judgment conditions (conditions 1 and 2) until they had been understood and checked for plausibility. The differences between the pseudoword condition (condition 3 ) and the plausibility judgment conditions (conditions 1 and 2) may thus be due to additional duration of processing of anomalous sentences in the plausibility judgment conditions (conditions 1 and 2) than of sentences containing pseudowords in the pseudoword condition (condition 3). If so, the results would not be relevant to the localization of semantic processing in sentence comprehension.

The result of the a priori comparisons that we will chiefly focus on is the increase in $\mathrm{rCBF}$ in Broca's area in the subtraction of the right-branching plausibility condition (condition 2) from the center-embedded plausibility condition (condition 1). The stimuli in the right-branching and center-embedded plausibility conditions differed in syntactic structure, and were controlled for length, semantic content (scenario), lexical items, point of anomaly, discourse referential presuppositions, and other potentially confounding factors, and were counterbalanced for order of presentation. In addition, subjects performed the same task on the two sets of materials, and the task required that sentences be structured and understood. Therefore, unlike the RT and rCBF differences between the pseudoword condition (condition 3) and the plausibility judgment conditions (conditions 1 and 2), it is unlikely 
that strategies unrelated to sentence processing caused the differences between the right-branching and center-embedded plausibility conditions (conditions 1 and 2). The region in which there is a difference in $\mathrm{rCBF}$ in these two conditions is thus a good candidate for an area of the brain involved in syntactic processing or related functions.

Before discussing possible processes that could have led to this increase in $\mathrm{rCBF}$, we will consider two issues that are relevant to the interpretation of this result. First, the increase in $\mathrm{rCBF}$ was found in a plausibility judgment task, and might be specific to this task. Second, the sentences were presented visually rather than auditorily, and the results may only arise with visual presentation. However, there are good reasons to believe that the results obtained in this study reflect aspects of processing that are not unique to plausibility judgments or visually presented sentences. Results with a variety of tasks that use both visual and auditory presentation are in agreement both with respect to the fact that center-embedded sentences are more difficult than right-branching sentences and with respect to the locus of the increase in processing (phoneme monitoring: Frauenfelder, Segui, \& Mehler, 1980; dual task performance: Wanner \& Maratsos, 1978; self-paced reading: King \& Just, 1990; speeded acceptability judgments for visually presented whole sentences both in isolation and with a concurrent memory load: Waters et al., 1987). This suggests that there are aspects of processing these sentence types that are common to many tasks and to the visual and auditory modalities of presentation. An additional point regarding the possibility that the results are determined by the visual presentation mode is that the location of the activation is in an area not known to be related to visual processing, but that is strongly implicated in language processing. Taken together, these considerations make it likely that the increase in $\mathrm{rCBF}$ reflects operations common to the processing of both visually and auditorily presented sentences of these types in many tasks. In our discussion, we shall concentrate on psycholinguistic and cognitive factors that could be responsible for the effect of sentence type. However, future research will have to determine whether the effect is in fact due to these central factors or is tied to operations connected to the visual processing of the sentences or the plausibility judgment task.

We may also consider and reject as unlikely several accounts of the differences between the right-branching and center-embedded plausibility conditions that are not directly related to assigning syntactic structure or related functions. Because reaction times were slower for center-embedded sentences than right-branching sentences, one consideration that can be discounted is that the greater $\mathrm{rCBF}$ in center-embedded than right-branching relative clause constructions was due to a greater rate of presentation for center-embedded than right-branching sentences. (For a discussion of the effects of different rates of presentation on PET scans, see Raichle, 1991; and Raichle, Fiez, Videen, MacLeod, Pardo, Fox, \& Petersen, in press). Another 
possibility is that the increase in $\mathrm{rCBF}$ seen in pars opercularis in the subtraction of the right-branching from the center-embedded plausibility condition might be due to a higher level of arousal in the center-embedded plausibility condition (condition 1) than in the right-branching plausibility condition (condition 2). Though it is possible that arousal contributes to the increased rCBF seen in pars opercularis in this subtraction, it seems unlikely that increased arousal can explain this increase completely. Increases in cingulum rCBF attributed to arousal do not always show corresponding increased rCBF in left frontal structures (e.g., Posner et al., 1988); rather, left frontal structures tend to show increased $\mathrm{rCBF}$ in certain language tasks. We therefore suggest that the increase in $\mathrm{rCBF}$ in pars opercularis seen in the subtraction of the right-branching plausibility condition from the center-embedded plausibility condition is at least partially due to more specific cognitive processes that simply arousal.

One possibility is that the difference between the center-embedded and right-branching plausibility conditions (conditions 1 and 2) reflects differences in the particular parsing operations involved in assigning the structure of center-embedded and right-branching sentences. Though many of these operations are the same in the two sentence types, there is evidence that there is a greater memory load associated with parsing center-embedded than right-branching sentences (Marcus, 1980; Berwick \& Weinberg, 1984; King \& Just, 1990). One possibility is that the increase in rCBF found in Broca's area in subtracting the right branching plausibility condition from the center-embedded plausibility condition reflects changes in neuronal activity associated with this increased memory load, and that it is therefore due to a highly specific psycholinguistic difference in processing these sentence types.

Another possibility is that the increase in rCBF in Broca's area associated with the subtraction of the right branching plausibility condition from the center-embedded plausibility condition is due to increases in the activity of a general verbal working memory capacity. The difference between this view and the first account is that this view maintains that the memory system that is more engaged by center-embedded than by right-branching sentences is one that is used in a wide variety of verbal tasks, not one that is completely dedicated to syntactic processing (Just \& Carpenter, 1992). The resolution of this issue is tied to the result of an ongoing debate over the role of a general verbal working memory system in syntactic processing in sentence comprehension (Miyake, Carpenter \& Just, 1994, 1995; Waters, Caplan \& Rochon, 1994; Caplan \& Waters, 1995; Waters \& Caplan, in press).

Another possibility is that rehearsal might be more engaged in processing center-embedded than right-branching structures. This account is attractive because we have the subjective feeling that we sometimes rehearse these sentences in making judgments about their plausibility, and because some PET results have found an increase in $\mathrm{rCBF}$ in Broca's area that may be due 
to rehearsal (Zatorre et al., 1992). However, before accepting the view that increased rehearsal accounts for the increased $\mathrm{rCBF}$ in pars opercularis, we should note that experimental psycholinguistic results suggest that rehearsal is not used more in making plausibility judgments about center-embedded than right-branching sentences (Waters et al., 1987), and that a patient with a severe impairment in rehearsal has been shown to understand center-embedded sentences very well (Waters, Caplan, \& Hildebrandt, 1991). Thus, although rehearsal may involve Broca's area, the differences between the center-embedded and right-branching plausibility conditions may not be due to differences in the degree of rehearsal involved in these two conditions.

A final possibility that we can suggest is that the increase in $\mathrm{rCBF}$ that occurred in pars opercularis in the subtraction of the right-branching plausibility judgment condition from the center-embedded plausibility judgment condition is associated with a variety of syntactic processes, not just these two sentence types. It may be that comparison of rCBF associated with processing passive versus active sentences, and other types of sentences, would also lead to similarly localized increases in $\mathrm{rCBF}$. On this account, a variety of factors that make one sentence more syntactically complex would engage operations that are principally located in Broca's area.

The finding that a part of Broca's area increases its $\mathrm{rCBF}$ when a syntactic structure is varied provides evidence that this region of the brain is involved in syntactic processing (or related processing, as discussed above). ${ }^{4}$ However, exactly what role this region plays in the neural system that is involved in this processing remains to be determined. The increase in electrophysiological activity that is associated with increases in $\mathrm{rCBF}$ in a region of the brain may reflect either excitatory or inhibitory neuronal activity. Moreover, either excitatory or inhibitory neuronal activity could be associated with a particular mental function being carried out in a particular brain region, or with that region regulating another area in which these computations are performed. Thus, the present data are consistent with the view that a part of Broca's area actively participates in the processes associated with syntactic analysis, or that it is involved in the control of a complex neural system in which these computations are performed. In addition, PET may not be capable of identifying all changes in $\mathrm{rCBF}$ in all the regions of the brain that are associated with a particular function. It is possible, for example, that PET only detects those areas in which these changes are greatest. Thus, the highly localized findings putatively pertaining to syntactic processing in this study could be reconciled with the neuropsychological finding of variability in the relationship of lesion sites to syntactic processing deficits in sentence comprehension (Caplan et al., 1985; Caplan, Hildebrandt, \& Makris, in press).

\footnotetext{
${ }^{4}$ Individual differences in brain morphology make it likely that there is more variance in the localization of this increase in $\mathrm{rCBF}$ than indicated in our analyses. However, the localization is directly within the pars opercularis in the Taliarach and Szikla (1967) atlas, and the jackknife analyses increase confidence in the localization of this increased $\mathrm{rCBF}$ in this region.
} 
In summary, the results of these studies indicate that the PET technique of functional neuroimaging is capable of providing data relevant to the localization of aspects of the sentence comprehension process. The most specific result was that an increase in rCBF occurred in part of Broca's area when the processing of two closely related sentence types-sentences with center-embedded and right-branching clauses - was compared. The results provide evidence supporting the role of a portion of Broca's area in the assignment of syntactic structure in sentence comprehension, or in operations associated with this process. Additional studies are needed to clarify both the exact processes that are involved in this comparison and the exact role that Broca's area plays in the neural system that underlies this aspect of sentence comprehension.

\section{REFERENCES}

Alpert, N. M., Berdichevsky, D., Weise, S., Tang, J., \& Rauch, S. L. 1993. Sterotactic transformation of PET scans by nonlinear least squares. In K. Uemura et al. (Eds.), Quantification of brain function: Tracer kinetics and image analysis in brain PET. Amsterdam: Elsevier Science Publishers. Pp. 459-463.

Berwick, R. C., \& Weinberg, A. 1984. The grammatical basis for linguistic performance: Language use and acquisition. Cambridge, MA: MIT Press.

Bookheimer, S. Y., Zeffiro, T. A., Gallard, W., \& Theodore, W. 1993. Regional cerebral blood flow changes during the comprehension of syntactically varying sentences. Neuroscience Society Abstracts, 347.5, 843.

Caplan, D. 1987. Neurolinguistics and linguistic aphasiology. Cambridge, UK: Cambridge Univ. Press.

Caplan, D. 1992. Language: Structure, processing and disorders. Cambridge, MA: MIT Press.

Caplan, D., Baker, C., \& Dehaut, F. 1985. Syntactic determinants of sentence comprehension in aphasia. Cognition, 21, 117-175.

Caplan, D., \& Futter, C. 1986. Assignment of thematic roles to nouns in sentence comprehension by an agrammatic patient. Brain and Language, 27, 117-134.

Caplan, D., Hildebrandt, H., \& Waters, G. S. 1994. Interaction of verb selectional restrictions, noun animacy, and syntactic form in sentence processing. Language and cognitive processes, 9, 549-585.

Caplan, D., Hildebrandt, H., and Makris, N. Location of lesions in stroke patients with deficits in syntactic processing in sentence comprehension, under review.

Caplan, D., \& Hildebrandt, N. 1988. Disorders of syntactic comprehension. Cambridge, MA: MIT Press (Bradford Books).

Caplan, D., \& Waters, G. S. 1995. Aphasic disturbances of syntactic comprehension and working memory capacity. Cognitive Neuropsychology, 12, 637-649.

Caramazza, A., \& Zurif, E. B. 1976. Dissociation of algorithmic and heuristic processes in language comprehension: Evidence from aphasia. Brain and Language, 3, 572-582.

Chertkow, H., Bub, D., Waters, G. S., Evans, A., Whitehead, V., \& Hosein, C. 1993. Separate effects of instructions and stimuli on cerebral blood flow on $\mathrm{O}^{15}$ tomographic transmission. Neurology, 43, A189.

Damasio, A. R. 1992. Aphasia. New England Journal of Medicine, 326, 531-539.

Demonet, J. F., Chollet, F., Ramsay, S., Cardebat, D., Nespoulous, J. L., Wise, R., Rascol, A., \& Frackowiak, R. 1992. The anatomy of phonological and semantic processing in normal subjects. Brain, 115, 1753-1768.

Demonet, J. F., Wise, R., \& Frackowiak, R. S. J. 1993. Language functions explored in normal subjects by positron emission tomography: A critical review. Human Brain Mapping, 1, 39-47. 
Fodor, J. 1983. Modularity of mind. Cambridge, MA: MIT Press.

Frauenfelder, U., Segui, J., \& Mehler, J. 1980. Monitoring around the relative clause. Journal of Verbal Learning and Verbal Behavior, 19, 328-337.

Friston, K. J., Frith, C. D., Liddle, P. F., \& Frackowiak, R. S. J. 1991. Comparing functional (PET) images: The assessment of significant change. Journal of Cerebral Blood Flow and Metabolism, 11, 690-699.

Frith, C. D., Friston, K. J., Liddle, P. F., \& Frackowiak, R. S. J. 1991. A PET study of word finding. Neuropsychologia, 29(12), 1137-1148.

Just, M. A., \& Carpenter, P. A. 1992. A capacity of theory of comprehension: Individual differences in working memory. Psychological Review, 99(1), 122-149.

King, J., \& Just, M. A. 1991. Individual differences in sentence processing: The role of working memory. Journal of Memory and Language, 30, 580-602.

Linebarger, M. C., Schwartz, M. F., \& Saffran, E. M. 1983. Sensitivity to grammatical structure in so-called agrammatic aphasics. Cognition, 13, 361-392.

Marcus, M. P. 1980. A theory of syntactic recognition for natural language. Cambridge, MA: MIT Press.

Mazoyer, B. M., Tzourio, N., Frak, V., Syrota, A., Murayama, N., Levrier, O., Salamon, G., Dehaene, S., Cohen, L., \& Mehler, J. 1993. The cortical representation of speech. Journal of Cognitive Neuroscience, 5(4), 467-479.

Mesulam, M. 1990. Large-scale neurocognitive networks and distributed processing for attention, language, and memory. Annals of Neurology, 28(5), 597-613.

Miyake, A., Carpenter, P. A., \& Just, M. A. 1994. A capacity approach to syntactic comprehension disorders: Making normal adults perform like aphasic patients. Cognitive Neuropsychology, 11, 671-717.

Miyake, A., Carpenter, P. A., \& Just, M. A. 1995. Reduced resources and specific impairments in normal and aphasic sentence comprehension. Cognitive Neuropsychology, 12, 651679.

Mohr, J. P., Pessin, M. S., Finkelstein, S., Funkenstein, H. H., Duncan, G. W., \& Davis, K. R. 1978. Broca aphasia: Pathologic and clinical. Neurology, 28, 311-324.

Mostellar, F., \& Tukey, J. 1977. Data analysis and regression. Reading, MA: AddisonWesley.

Munte, T. F., Heinze, H. J., \& Mangun, G. R. 1993. Dissociation of brain activity related to syntactic and semantic aspects of language. Journal of Cognitive Neuroscience, 5(3), 335344.

Neville, H., Nicol, J. L., Barss, A., Forster, K. I., \& Garrett, M. F. 1991. Syntactically based sentence processing classes: Evidence from event-related brain potentials. Journal of Cognitive Neuroscience, 3(2), 151-165.

Paulesu, E., Frith, C. D., \& Frackowiak, R. S. J. 1993. The neural correlates of the verbal component of working memory. Nature, 362, 342-345.

Petersen, S. E., Fox, P. T., Posner, M. I., Mintun, M., \& Raichle, M. E. 1988. Positron emission tomographic studies of the cortical anatomy of single-word processing. Nature, 331, 585589.

Petersen, S. E., Fox, P. T., Posner, M. I., Mintun, M., \& Raichle, M. E. 1989. Positron emission tomographic studies of the processing of single words. Journal of Cognitive Neuroscience, 1(2), 153-170.

Petersen, S. E., Fox, P. T., Snyder, A. Z., \& Raichle, M. E. 1990. Activation of extrastriate and frontal cortical areas by visual words and word-like stimuli. Science, 249, 10411044.

Posner, M., Petersen, S., Fox, P., \& Raichle, M. 1988. Localization of cognitive operations in the human brain. Science, 240, 1627-1632.

Raichle, M. E. 1991. Memory mechanisms in the processing of words and word-like symbols. In D. J. Chadwick \& J. Whelan (Eds.), CIBA Foundation Symposium: Exploring Brain Functional Anatomy with Positron Tomography, Vol. 163 London: Wiley. Pp. 198217. 
Raichle, M. E., Fiez, J. A., Videen, T. O., MacLeod, A. K., Pardo, J. V., Fox, P. T., \& Petersen, S. E. 1994. Practice-related changes in human brain functional anatomy during non-motor learning. Cerebral Cortex, 4, 8-26.

Rosler, F., Putz, P., Friederici, A., \& Hahne, A. 1993. Event-related brain potentials while encountering semantic and syntactic constraint violations. Journal of Cognitive Neuroscience, 5(3), 345-362.

Schwartz, M. F., Linebarger, M. C., Saffran, E. M., \& Pate, D. S. 1987. Syntactic transparency and sentence interpretation in aphasia. Language and Cognitive Processes, 2(2), 85-113.

Selnes, O. A., Knopman, D. S., Niccum, N., Rubens, A. B., \& Larson, D. 1983. Computed Tomographic scan correlates of auditory comprehension deficits in aphasia: A prospective recovery study. Annals of Neurology, 13, 558-566.

Sergent, J., Zuck, E., Levesque, M., \& MacDonald, B. 1992. Positron Emission Tomography study of letter and object processing: Empirical findings and methodological considerations. Cerebral Cortex, 2, 68-80.

Shallice, T. 1988. From neuropsychology to mental structure. Cambridge, UK: Cambridge Univ. Press.

Talairach, J., \& Szikla, Z. 1967. Atlas of sterotaxic anatomy of the telencephalon. Paris: Masson \& Cie.

Tramo, M. J., Baynes, K., \& Volpe, B. T. 1988. Impaired syntactic comprehension and production in Broca's aphasia. Neurology, 38, 95-98.

Tyler, L. 1985. Real-time comprehension processes in agrammatism: A case study. Brain and Language, 26, 259-275.

Vanier, M., \& Caplan, D. 1990. CT correlates of agrammatism. In L. O. L. Menn \& H. Goodglassa (Eds.), A cross-language study of agrammatism. New York: Benjamin.

Wanner, E., \& Maratsos, M. 1978. An ATN approach to comprehension. In G. M. M. Halle \& J. Bresnan (Eds.), Linguistic theory and psychological reality. Cambridge, MA: MIT Press. Pp. 119-161.

Waters, G., \& Caplan, D. The capacity theory of sentence comprehension: A reply to Just and Carpenter. Psychological Review, In press.

Waters, G., Caplan, D., \& Hildebrandt, N. 1987. Working memory and written sentence processing. In M. Coltheart (Ed.), Attention and performance XII: The psychology of reading. London: Erlbaum. Pp. 531-555.

Waters, G. S., Caplan, D., and Hildebrandt, N. 1991. On the structure and function role of auditory-verbal short-term memory in sentence comprehension: A case study. Cognitive Neuropsychology, 2, 81-126.

Waters, G. S., Caplan, D., Rochon, E., and Waters, G. 1995. Processing resources and sentence comprehension in patients with Alzheimer's disease. Cognitive Neuropsychology,12, 130.

Wise, R., Chollet, F., Hadar, U., Friston, K., Hoffner, E., \& Frackowiak, R. 1991. Distribution of cortical neural networks involved in word comprehension and word retrieval. Brain, 114, 1803-1817.

Zatorre, R. J., Evans, A. C., Meyer, E., \& Gjedde, A. 1992. Lateralization of phonetic and pitch discrimination in speech processing. Science, 256, 846-849.

Zurif, E., Swinney, D., Prather, P., Solomon, J., \& Bushell, C. 1993. An on-line analysis of syntactic processing in Broca's and Wernicke's aphasia. Brain and Language, 45, 448463. 